\title{
Spontaneous intracranial hypotension complicated by diffuse cerebral edema and episodes of severely elevated intracranial pressure: illustrative case
}

\author{
Jeffrey P. Turnbull, BS, and Vittorio M. Morreale, MD \\ Department of Neurosurgery, Henry Ford Macomb Hospital, Clinton Township, Michigan
}

BACKGROUND Spontaneous intracranial hypotension $(\mathrm{SIH})$ is a well-documented condition that typically follows a defined clinical course. Previously published studies describing the pathophysiology of SIH have demonstrated extensive evidence of low intracranial pressure (ICP) driving the clinical features of the condition. Through lumbar puncture and use of intracranial monitoring devices, however, both low and normal cerebrospinal fluid (CSF) pressures have been documented. This report outlined and discussed the unique finding of elevated ICP associated with clinical features of SIH.

OBSERVATIONS Here, the authors presented a case of a patient with spontaneous spinal CSF leak who developed tonsillar herniation, cerebral edema, and subsequent episodes of elevated ICP. Although more diverse presentations of SIH are being reported, the authors believed the case to be unique because SIH was accompanied by elevated ICP.

LESSONS This case adds to the growing body of literature surrounding SIH by demonstrating that patients can develop elevated CSF pressures associated with acute encephalopathy.

https://thejns.org/doi/abs/10.3171/CASE21118

KEYWORDS spinal CSF leak; orthostatic headache; elevated intracranial pressure; spontaneous intracranial hypotension; tonsillar herniation

Spontaneous intracranial hypotension $(\mathrm{SIH})$ represents a clinical syndrome characterized by low pressure headaches resulting from spontaneous leakage of cerebrospinal fluid (CSF) into the adjacent epidural space or adjacent paraspinal soft tissues. Although its clinical and radiological recognition have been increasingly better understood and more thoroughly documented, SIH remains a rare phenomenon, with an estimated reported incidence of 5 per 100,000 per year. ${ }^{1}$

This disorder can manifest in different ways and is associated with various symptoms and levels of morbidity. Most patients with $\mathrm{SIH}$ follow a mild clinical course and present with an orthostatic headache. ${ }^{2-5}$ The quality of the headache may vary in intensity and location but typically presents within minutes of assuming an upright position and ceases shortly after recumbency. ${ }^{4}$ This headache can be debilitating and, in severe cases, is described as "the worst headache of one's life" (i.e., thunderclap headache)., ${ }^{4,5}$ Other documented symptoms include posterior neck pain or stiffness, nausea, vomiting, auditory disturbances, and cranial nerve palsies. ${ }^{1-5}$
Although rare, a growing body of literature has described the potentially severe manifestations associated with $\mathrm{SIH}$, including acute encephalopathy, coma, cerebral hemorrhage, and subdural hematomas. $^{6-9}$ Although the etiology of $\mathrm{SIH}$ is still relatively unknown, instability or structural defects in the dural membrane are largely regarded to be associated with CSF leakage. ${ }^{2,5}$ Two inciting mechanisms that have been previously established are a history of mild physical trauma preceding symptoms and an underlying connective tissue disorder. ${ }^{1,10}$ Upon surgical exploration, leaking meningeal diverticulum or dural tears have been documented. ${ }^{1-3}$ In a significant percentage of patients with $\mathrm{SIH}$, however, an appreciable source of meningeal structural disruption was never located., ${ }^{2,3}$

Radiological findings associated with SIH have been well documented, with some features becoming pathognomonic for the disease. Gadolinium-enhanced cranial magnetic resonance imaging (MRI) demonstrates these characteristic findings: (1) diffuse symmetrical pachymeningeal enhancement, (2) cerebral edema and

ABBREVIATIONS CSF = cerebrospinal fluid; CT = computed tomography; DSM = digital subtraction myelography; EBP = epidural blood patch; ICP = intracranial pressure; $\mathrm{MRI}=$ magnetic resonance imaging; $\mathrm{SIH}=$ spontaneous intracranial hypotension.

INCLUDE WHEN CITING Published December 6, 2021; DOI: 10.3171/CASE21118.

SUBMITTED March 1, 2021. ACCEPTED September 9, 2021.

(C) 2021 The authors, CC BY-NC-ND 4.0 (http://creativecommons.org/licenses/by-nc-nd/4.0/). 
effacement of sulci and basilar cisterns, (3) sagging of the brain and cerebellar tonsil ectopia, and (4) engorgement of venous structures. $^{1,4,11,12}$ If further localization of the leak's source is required, thin-slice axial computed tomography (CT) myelography or radionucleotide cisternography traditionally has been used. ${ }^{1,3,4}$ As the name suggests, intracranial hypotension is associated with low CSF pressures, typically less than $<6 \mathrm{~cm} \mathrm{H}_{2} \mathrm{O}^{1,10}$ Although low pressures are a common finding, variable pressure readings have been documented. They can range from negative values to consistently normal CSF pressures. To the authors' knowledge, there has yet to be a report of severely elevated intracranial pressure (ICP) readings associated with $\mathrm{SIH}$. In this case report, we describe a patient who possessed characteristic clinical and radiological findings consistent with intracranial hypotension but uniquely demonstrated severely elevated ICP readings subsequent to presentation.

\section{Illustrative Case}

\section{Initial Evaluation}

Our patient is a 59-year-old woman who presented to the emergency department with altered mental status after falling in her home. She had a past medical history of Crohn's colitis and hypothyroidism, and her previous surgeries included a colectomy with colostomy. She was being treated with ustekinumab (Stelara), azathioprine, Synthroid, and allopurinol. Upon initial assessment, she was confused as to why she was in the hospital; however, within 24 hours of admission, she was able to provide a history. Regarding her fall, she reported no major trauma to her head and no loss of consciousness, and she denied a previous history of falls. Initial neurological examination was unremarkable and mentation was intact. Approximately 48 hours after admission, the patient developed persistent orthostatic headaches, which were reported as a nonspecific holocephalic pressure headache associated with nausea and vomiting. She referred to them as the worst headaches of her life. More specifically, the headaches became worse within $30 \mathrm{sec}-$ onds of rising from a supine position and almost completely resolved by lying flat. She denied a previous history of similar headaches. In addition, she denied photophobia, phonophobia, changes in vision, hearing loss, dysarthria, dysphagia, weakness, numbness, or paresthesias.

Noncontrast axial CT demonstrated diffuse cerebral edema with effacement of the sulci and basilar cisterns and a small subdural hematoma scattered along the falx cerebri (Fig. 1). Gadoliniumenhanced brain MRI redemonstrated diffuse cerebral edema. Sagittal views showed cerebellar tonsillar ectopia and evidence of descending transtentorial herniation. They also showed diffuse pachymeningeal and leptomeningeal enhancement (Fig. 2). Noncontrast sagittal MRI of the thoracic spine demonstrated a possible epidural fluid collection near the mid- to upper thoracic spine but was otherwise unremarkable. Lumbar puncture was not performed because of risk of exacerbating herniation.

\section{Clinical Course, Working Diagnosis, and First Intervention}

Initial working diagnosis was SIH resulting from a CSF leak. The patient was initially treated for decreased ICP with fluid management and analgesics. On day 4 of admission, the patient became lethargic and confused. She was noted to have sun-setting eyes (downward gaze) and her pupils were sluggish to respond; however, cranial nerves were grossly intact. Upper and lower extremity strength was $4 / 5$ bilaterally. Her neurological status rapidly declined,

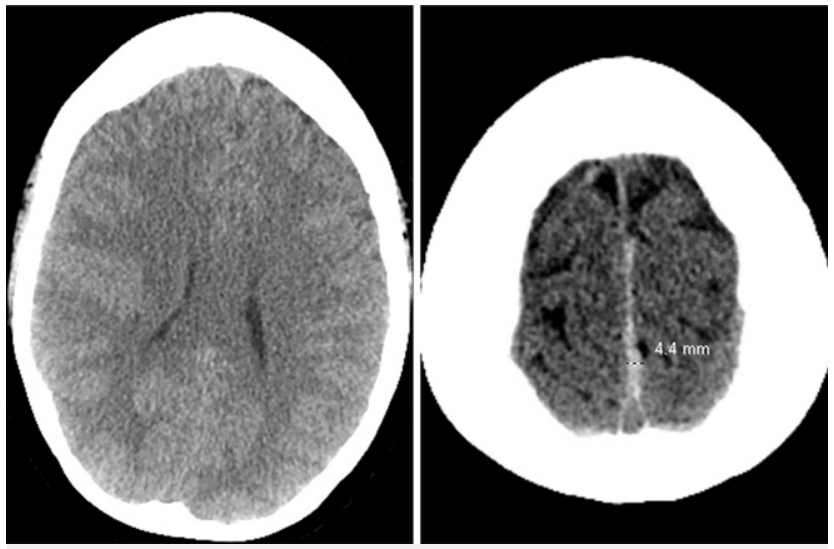

FIG. 1. Axial CT scans without contrast demonstrating diffuse cerebral edema with effacement of bilateral cerebral sulci (left) and small subdural hemorrhage along the cerebral falx (right).

and she became unresponsive, only being able to localize to pain. The patient was treated with 3\% hypertonic saline and mannitol to reduce cerebral edema and initially improved with these ICP-lowering treatments. Despite these interventions, however, she subsequently decompensated, becoming completely unresponsive and developing fixed and dilated pupils. Neurosurgical staff quickly placed an ICP monitor. The initial reading was $89 \mathrm{~mm} \mathrm{Hg}$. She was
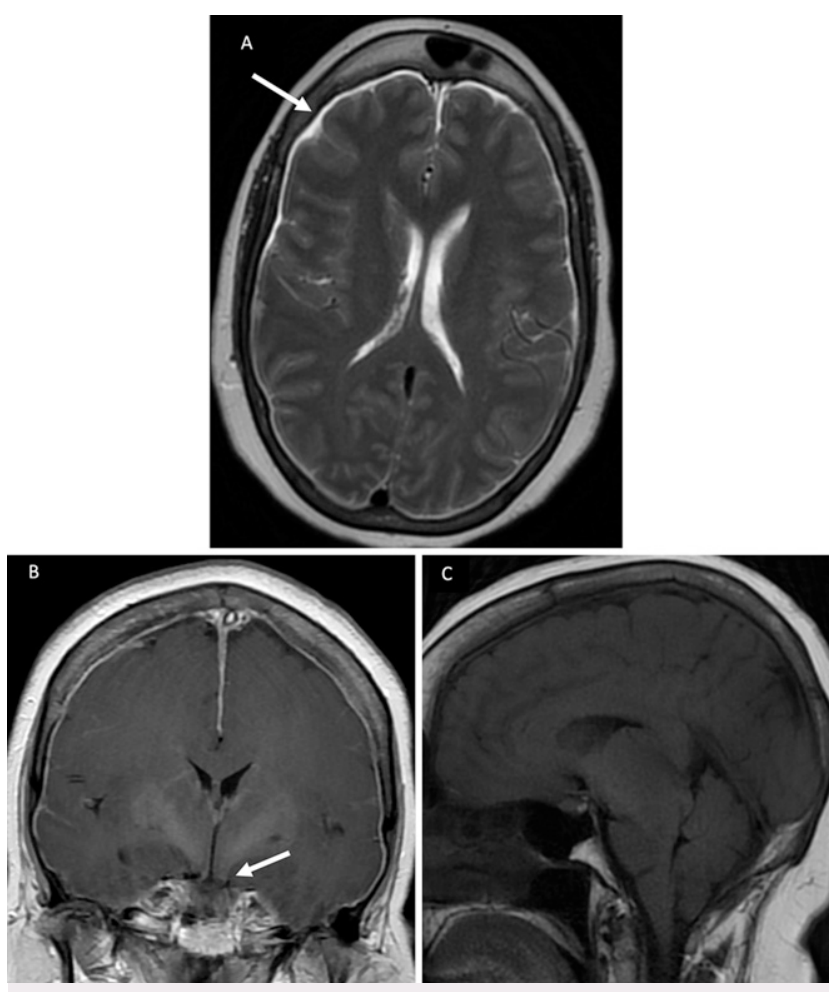

FIG. 2. MRI obtained shortly after presentation. A: Axial T2-weighted MRI showing increased subdural fluid (white arrow). B: Coronal T1weighted gadolinium-enhanced MRI revealing pachymeningeal enhancement and descending transtentorial herniation (white arrow). C: Sagittal T1-weighted MRI showing significant cerebellar tonsillar herniation. 
intubated and received hyperventilation, which dropped her ICP to $15 \mathrm{~mm} \mathrm{Hg}$. CT/CT angiography of her head was performed immediately. Fortunately, adequate blood flow to the brain was demonstrated and no acute changes were visible. An urgent decompressive suboccipital craniectomy with $\mathrm{C} 1$ laminectomy and duroplasty was subsequently performed. The patient was found to have significantly herniated cerebellar tonsils and bilateral contusions of the cerebellar hemispheres, consistent with acute herniation. The cerebellar tonsils were subsequently freed from restriction, CSF flow through the fourth ventricle was confirmed, and the posterior fossa appeared to be nicely decompressed. Lastly, an epidural blood patch (EBP) was placed roughly at the T2-T4 level using $20 \mathrm{~mL}$ of autologous blood.

\section{Reevaluation and Second Intervention}

The patient initially responded well during the postoperative period. Her neurological status improved dramatically. She became awake and alert, followed commands, and became conversant. She demonstrated no focal neurological deficits. She denied headache or dizziness, and ICP monitor readings were $<8 \mathrm{~mm} \mathrm{Hg}$. Elevation of the head of her bed was gradually introduced to evaluate for signs of orthostatic headaches. Approximately 2 days postoperatively, her mental status began to fluctuate between awake, alert, and oriented and stuporous and poorly responsive to vigorous stimulation. Her ICP normalized. When able, she mentioned that the headaches had returned and reported being significantly nauseated. Similar to the previous presentation, she reported increased severity of the headaches with elevation of the bed above $15^{\circ}$ and when sitting up for meals. On neurological examination, there was concern that she had developed new-onset left upper extremity weakness. Because these were signs that the initial blood patch did not take, two more attempts of EBP placement were made at levels T5-T6 and T3-T4, but symptoms persisted. At this time, a CT myelogram demonstrated small amounts of contrast exiting multiple neural foramina in the upper thoracic spine. It was most pronounced at the T3-T4 and T4-T5 levels on the right (Fig. 3).

\section{Reevaluation and Final Intervention}

Because of repeated failure of EBP placement, a bilateral T3-T6 exploratory laminectomy was performed for CSF leak repair. During the procedure, several abnormalities were noted. At T3-T4 on the left, there was significant swelling of the nerve root consistent with a dural defect and CSF cyst formation, but no active CSF leakage was observed. At T4-T5 on the left, marked thinning of the dura at the axilla of the nerve root was present, but again, no CSF leak was observed. Valsalva maneuver did not induce CSF leak from these sites. At the level of T5-T6 on the left, the dura was markedly attenuated and appeared translucent, allowing for visualization of the underlying nerve roots. Here, general CSF seepage was observed; however, no specific defect was noted. Because of a lack of observable defect to suture, the nerve root was packed with Floseal (Baxter Healthcare) and powdered Surgicel (Ethicon). It was then wrapped in a layer of DuraGen (Integra Lifesciences), followed by a layer of DuraSeal (Covidien). Subsequent CSF leakage was not appreciated intraoperatively.

\section{Posttreatment Course}

During the postoperative period, the patient recovered well and was awake and alert and oriented to self and her situation. She reported that her headaches were decreasing in frequency and severity and denied experiencing any focal neurological deficits.

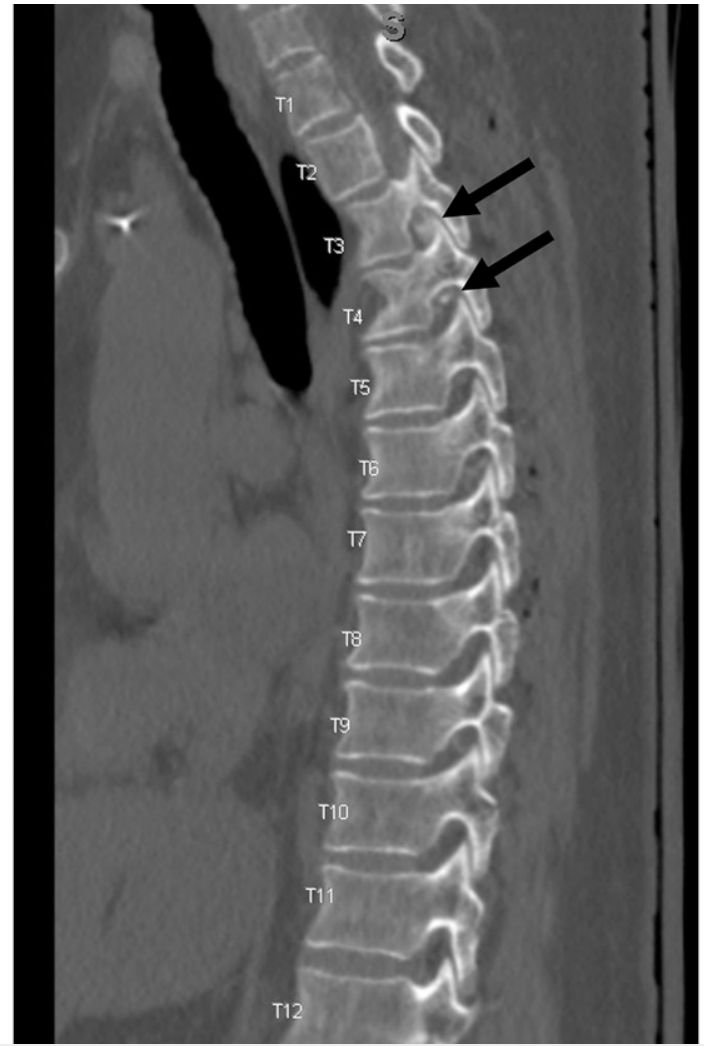

FIG. 3. Sagittal contrast-enhanced CT myelogram of the thoracic spine demonstrating contrast material leakage most pronounced at T3-T4 and T4-T5 neural foramina (black arrows).

Her ICP monitor repeatedly demonstrated readings $<10 \mathrm{~mm} \mathrm{Hg}$, and the ICP bolt was subsequently removed. The patient was then discharged to an in-patient rehabilitation facility, where she completed her rehabilitative course without incident or recurrence of symptoms. At her 5-month postoperative visit, the patient reported complete resolution of her headaches and associated symptoms, with restoration of her previous baseline functioning. A follow-up MRI with and without contrast demonstrated reelevation of midline structures to normal positioning and resolution of both pachymeningeal enhancement and the frontal subdural hematoma.

\section{Discussion}

The pathophysiology regarding low pressure headaches has been well defined. Persistent drainage from CSF leaks creates CSF hypovolemia and reduced pressure in the intrathecal space. This alteration in fluid dynamics exerts a "pull-down" phenomenon in which absent or reduced CSF buoyancy allows for diffuse sagging of intracranial structures overlying the cranial base and posterior cranial fossa. ${ }^{3}$ This condition is most notably observed as inferior displacement of the cerebellar tonsils, flattening of the pons against the clivus, and effacement of basal cisterns, ${ }^{3,4,11}$ all of which were demonstrated in our patient. The myriad of clinical symptoms experienced by patients with a CSF leak is likely a result of the combination of this diminished CSF buoyancy and the caudally displacing effects of gravity. Direct articulation of pressure-sensitive neural structures along the cranial floor and posterior fossa creates 
compressive or traction-like stress, eliciting headaches, nausea, vomiting, neurological deficits, and potentially impaired consciousness. ${ }^{3,5,6} \mathrm{~A}$ potential result of this associated brain sagging that has not yet been observed is acute deterioration in the setting of elevated ICP. On day 4 of admission, our patient developed prominent features of elevated ICP: sun-setting eyes, excessive lethargy, and eventual obtundation, which was confirmed with placement of an ICP monitor demonstrating pressures between $80 \mathrm{~mm} \mathrm{Hg}$ and $90 \mathrm{~mm} \mathrm{Hg}$. Although the patient was quickly treated and stabilized, we must ask the question: In the setting of a syndrome characterized by low ICP, how did such severely elevated CSF pressures develop?

\section{Observations}

One explanation for this development could be attributed to the obstructive nature of cerebellar tonsillar herniation. Previous literature has reported rare but severe manifestations of $\mathrm{SIH}$ in which transtentorial herniation and subsequent caudal displacement of the cerebellar tonsils resulted in both acute cerebral infarction and coma. ${ }^{7,8}$ Although tonsillar herniation producing a noncommunicating hydrocephalus has yet to be documented in the setting of $\mathrm{SIH}$, its association has been clearly delineated in Chiari I malformation. $^{13,14}$ Whereas Chiari I malformation and SIH are completely separate entities, they both feature inferiorly displaced cerebellar tonsils, drawing mechanistic similarities. Hydrocephalus is a less common but well-known manifestation of Chiari I malformation. ${ }^{13}$ Although the underlying pathophysiology of this relationship has been previously debated, it is well accepted that overcrowding of the foramen magnum by neural structures in the posterior cranial fossa likely induces the rise in ICP. ${ }^{13}$ At this level, obstruction of pulsatile CSF movement may occur via compression of the fourth ventricle or blockage of flow from the cranial subarachnoid cisterns across the foramen magnum. ${ }^{13}$ These alterations in CSF flow dynamics can cause ICP to rise, prompting clinical manifestations of hydrocephalus. We suggest that a similar mechanism of physical obstruction could have led to the acute rise in ICP and subsequent deterioration in our patient. Interestingly, however, we believe our patient's tonsillar herniation from low CSF pressure was the underlying mechanism leading to an acute rise in ICP. In addition to this, we reason that caudal displacement of the patient's cerebellar tonsils led to compression of draining veins restricting venous outflow. This condition likely created a "snowball effect" that increased cerebral edema and, as a result, the ICP. We further hypothesize that this accumulated cerebral edema increased the intracranial volume such that the patient's ICP rose to an inflection point. On a pressure-volume relationship curve, this inflection point reflects a state in which any minimal increase in intracranial volume exponentially elevates ICP. In this case, we believe that the acute herniation of her cerebellar tonsils that obstructed pulsatile CSF outflow may have minimally increased intracranial volume. However, because she was at an inflection point, it resulted in a massive rise in ICP.

Another possibility that may have led to an acute rise in ICP is related to reflexive increase in cerebral blood flow from CSF depletion. Per the Monro-Kellie doctrine, the sum of three distinct intracranial volumes must remain constant in an intact skull, including intracranial blood volume, CSF volume, and volume of the brain tissue itself. ${ }^{15}$ Whereas the volume of brain tissue remains relatively fixed, derangements in flow of intracranial CSF or cerebral blood will produce compensatory adjustments to maintain ICP. ${ }^{15}$ Thus, in the setting of a CSF leak and diminished intracranial CSF volumes, compensatory vasodilatation of cerebral vessels and increased blood flow is to be expected. ${ }^{15}$ When chronic leakage of CSF occurs, prolonged reflexive venous congestion may reduce vessel compliance and impair cranial venous outflow. This impaired outflow may subsequently raise ICP. This phenomenon was previously proposed by Craven et al. to describe elevated mean ICP in patients with persistent CSF leak after spinal surgery. ${ }^{16}$

\section{Lessons}

Despite our patient's resolution of symptoms through effective surgical intervention, we believe review of this case affords us insight into possible means to optimize the care of future patients who present with suspected spinal CSF leak. During our patient's stay, several imaging modalities were used to visualize areas of possible CSF leak, including MRI and CT myelography. As reported in other SIH literature, these modalities are valuable tools in demonstrating CSF leakage but can be less helpful when localizing the specific level of CSF extravasation. The superior temporal resolution of digital subtraction myelography (DSM) in comparison to dynamic CT myelography has been previously demonstrated. A study by Hoxworth et al. demonstrated the effective use of DSM when localizing spinal CSF leaks. ${ }^{17}$ Previous studies have also shown that DSM is an effective means of localizing CSF leaks in patients with SIH associated with dural nerve root CSF to venous fistulae. Upon review of literature, Farb et al. categorized spinal CSF leaks into four types and discussed use of DSM as a means of classifying CSF leaks to optimize management. ${ }^{18}$ During our patient's treatment course, thoracic spinal MRI demonstrated possible epidural fluid collection in the upper and middle thoracic spine. Several attempts at placing EBPs at various levels of the thoracic spine were made under the assumption that it was likely the site of CSF extravasation. After multiple failed attempts at EBP placement, we elected to obtain a CT myelogram, which demonstrated contrast extravasation at multiple levels of the thoracic spine. Overall, our patient's management may have been more effectively optimized through early use of DSM.

$\mathrm{SIH}$ is becoming a more commonly recognized and diagnosed condition as a result of better-defined clinical and radiological criteria from expanding literature on the subject. As a result of its increasing recognition, $\mathrm{SIH}$ is more frequently considered when evaluating patients with persistent and refractory headaches. Although more diverse presentations of $\mathrm{SIH}$ are being reported, we believe our case is unique in that $\mathrm{SIH}$ was accompanied by elevated ICP. This case adds to the growing body of literature surrounding $\mathrm{SIH}$ by demonstrating that patients can develop elevated CSF pressures associated with acute encephalopathy. Although we attempted to offer potential insight into the underlying mechanisms driving this presentation, this topic requires further investigation.

\section{References}

1. Schievink WI. Spontaneous spinal cerebrospinal fluid leaks and intracranial hypotension. JAMA. 2006;295(19):2286-2296.

2. Schievink WI, Morreale VM, Atkinson JLD, Meyer FB, Piepgras DG, Ebersold MJ. Surgical treatment of spontaneous spinal cerebrospinal fluid leaks. J Neurosurg. 1998;88(2):243-246.

3. Schievink WI, Meyer FB, Atkinson JLD, Mokri B. Spontaneous spinal cerebrospinal fluid leaks and intracranial hypotension. J Neurosurg. 1996;84(4):598-605.

4. Mokri B, Piepgras DG, Miller GM. Syndrome of orthostatic headaches and diffuse pachymeningeal gadolinium enhancement. Mayo Clin Proc. 1997;72(5):400-413. 
5. Bell WE, Joynt RJ, Sahs AL. Low spinal fluid pressure syndromes. Neurology. 1960;10(5):512-521.

6. Beck CE, Rizk NW, Kiger LT, Spencer D, Hill L, Adler JR. Intracranial hypotension presenting with severe encephalopathy. Case report. J Neurosurg. 1998;89(3):470-473.

7. Chi N-F, Wang S-J, Lirng J-F, Fuh J-L. Transtentorial herniation with cerebral infarction and duret haemorrhage in a patient with spontaneous intracranial hypotension. Cephalalgia. 2007;27(3):279-282.

8. Schievink WI, Maya MM, Moser FG, Jean-Pierre S, Nuño M. Coma: a serious complication of spontaneous intracranial hypotension. Neurology. 2018;90(19):e1638-e1645.

9. de Noronha RJ, Sharrack B, Hadjivassiliou M, Romanowski CA. Subdural haematoma: a potentially serious consequence of spontaneous intracranial hypotension. J Neurol Neurosurg Psychiatry. 2003;74(6):752-755.

10. Nosik WA. Intracranial hypotension secondary to lumbar nerve sleeve tear. J Am Med Assoc. 1955;157(13):1110-1111.

11. Atkinson JLD, Weinshenker BG, Miller GM, Piepgras DG, Mokri B. Acquired Chiari I malformation secondary to spontaneous spinal cerebrospinal fluid leakage and chronic intracranial hypotension syndrome in seven cases. J Neurosurg. 1998;88(2):237-242.

12. Pannullo SC, Reich JB, Krol G, Deck MD, Posner JB. MRI changes in intracranial hypotension. Neurology. 1993;43(5):919-926.

13. Massimi L, Pennisi G, Frassanito P, Tamburrini G, Di Rocco C, Caldarelli M. Chiari type I and hydrocephalus. Childs Nerv Syst. 2019;35(10):1701-1709.

14. Buell TJ, Heiss JD, Oldfield EH. Pathogenesis and cerebrospinal fluid hydrodynamics of the Chiari I malformation. Neurosurg Clin N Am. 2015;26(4):495-499.

15. Mokri B. The Monro-Kellie hypothesis: applications in CSF volume depletion. Neurology. 2001;56(12):1746-1748.

16. Craven C, Toma AK, Khan AA, Watkins LD. The role of ICP monitoring in patients with persistent cerebrospinal fluid leak following spinal surgery: a case series. Acta Neurochir (Wien). 2016;158(9): 1813-1819.
17. Hoxworth JM, Trentman TL, Kotsenas AL, Thielen KR, Nelson KD, Dodick DW. The role of digital subtraction myelography in the diagnosis and localization of spontaneous spinal CSF leaks. AJR Am J Roentgenol. 2012;199(3):649-653.

18. Farb RI, Nicholson PJ, Peng PW, et al. Spontaneous intracranial hypotension: a systematic imaging approach for CSF leak localization and management based on MRI and digital subtraction myelography. AJNR Am J Neuroradiol. 2019;40(4):745-753.

\section{Disclosures}

The authors report no conflict of interest concerning the materials or methods used in this study or the findings specified in this paper.

\section{Author Contributions}

Conception and design: both authors. Acquisition of data: both authors. Analysis and interpretation of data: both authors. Drafting the article: both authors. Critically revising the article: both authors. Reviewed submitted version of manuscript: both authors. Approved the final version of the manuscript on behalf of both authors: Morreale. Administrative/technical/material support: Morreale. Study supervision: Morreale.

\section{Supplemental Information}

Previous Presentations

Portions of this work were presented as a poster at the Virtual AANS Annual Scientific Meeting, August 21-25, 2021.

\section{Correspondence}

Vittorio M. Morreale: Henry Ford Macomb Hospital, Clinton Township, Ml. vmorreale@sbcglobal.net. 